\title{
Integrated-Antenna Push-Pull Power Amplifiers
}

\author{
William R. Deal, Member, IEEE, Vesna Radisic, Member, IEEE, Yongxi Qian, Member, IEEE, \\ and Tatsuo Itoh, Life Fellow, IEEE
}

\begin{abstract}
In this paper, the integrated-antenna concept is applied to push-pull power amplifiers (PA's). In this approach, the antenna serves as an out-of-phase power combiner and tuned load for higher harmonics. This new architecture effectively has a near-zero loss output hybrid, and results in a high-efficiency PA. The first example is a narrow-band push-pull amplifier integrated with a dual-feed patch antenna. At an operating frequency of $2.5 \mathrm{GHz}$, a maximum measured power-added efficiency (PAE) of $55 \%$ is achieved. The second example is a broadband push-pull amplifier integrated with a dual-feed slot antenna amplifier operating at $2.46 \mathrm{GHz}$ has a peak PAE of $63 \%$, and PAE is better than $55 \%$ in an $8 \%$ bandwidth. Additionally, $48 \%$ PAE is achieved with code-division multiple-access modulation and adjacent-channel power ratio better than $-42 \mathrm{dBc}$ at a $1.25-\mathrm{MHz}$ offset.
\end{abstract}

Index Terms - CDMA, FET amplifier, integrated antenna, power-added efficiency, push-pull.

\section{INTRODUCTION}

$\mathbf{T}$ WE push-pull amplifier has shown excellent properties at lower frequencies where they are easily implemented by using n-p-n- and p-n-p-type bipolar junction transistor (BJT) devices. However, at microwave and millimeter-wave frequencies, FET devices are typically used with a hybrid or balun to combine the power of the devices. While capable of broad-band performance, good linearity and offering twice the output power compared to a single-ended amplifier, the losses in the output stage hybrid directly limits the practical efficiency of this class of amplifier at microwave and millimeter-wave frequencies [1]. For example, a combining loss of $0.5 \mathrm{~dB}$ will decrease overall power-added efficiency (PAE) of two singleend amplifiers operating at $60 \%$ to $53 \%$. Various push-pull amplifiers have been presented in the literature [2]-[4].

Active devices have been directly integrated with the antenna platform to help solve many problems, including quasioptical amplifiers and mixers, transceivers, and frequency doublers [5]-[8]. Recently, the active integrated-antenna concept has been applied to tuned high-efficiency power amplifiers (PA's) [9]-[11]. This approach has the advantage of placing the PA directly at the antenna platform with minimal matching and interconnects. This leads to lower output losses and a compact transmitter front-end, critical for the stringent requirements of today's wireless systems. Increased efficiency

Manuscript received November 24, 1998. This work was supported by the Army Research Office Multidisciplinary Research Initiative under Contract DAAH04-96-1-0005, and by Rockwell International under a MICRO project.

W. R. Deal, Y. Qian, and T. Itoh are with the Department of Electrical Engineering, University of California at Los Angeles, Los Angeles, Los Angeles, CA 90095 USA.

V. Radisic is with HRL Laboratories, Malibu, CA 90265-4799 USA.

Publisher Item Identifier S 0018-9480(99)06078-0.

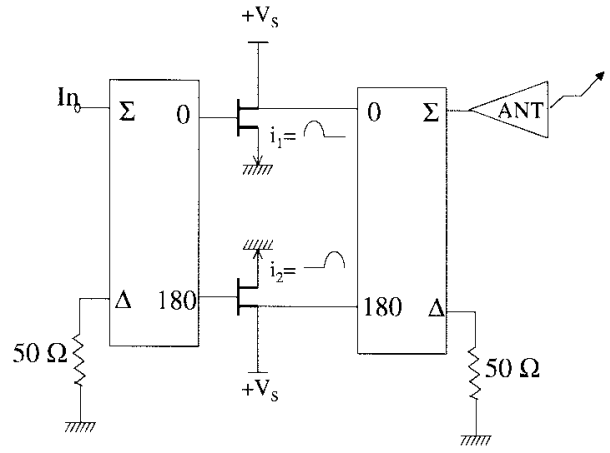

(a)

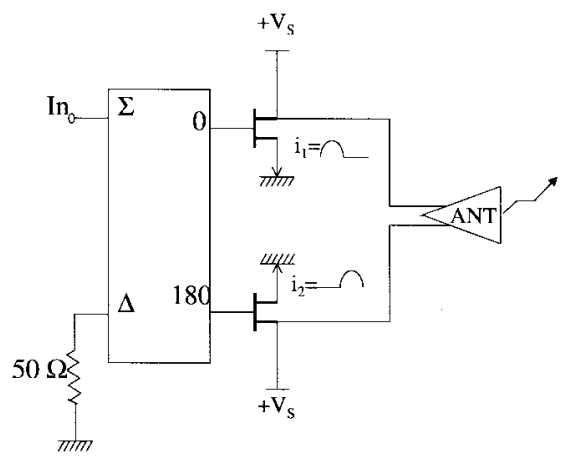

(b)

Fig. 1. Architecture of: (a) conventional and (b) integrated-antenna push-pull front-end.

reduces heat-sink size and increases battery life. Additionally, the whole transmitter can be fabricated on the same substrate as the antenna, leading to a compact and lightweight solution. In this paper, we present a new push-pull architecture, which utilizes the active integrated-antenna concept. The antenna acts as an out-of-phase combiner for the fundamental frequency and as a tuned load for higher harmonics, in addition to its original function of radiator.

The conventional push-pull architecture is shown in Fig. 1(a). The MESFET transistors are biased as class B (at pinchoff voltage). The input power is split and fed antiphase to the two FET's through a $180^{\circ}$ hybrid. The resulting current form at the output of the two MESFET consists of two antiphase half-sinusoids. Fourier analysis of the current waveform at the drain of each device reveals that they are composed of a dc term, a fundamental term, and a series of even harmonics

$$
\mathbf{i}_{d 1}=\mathbf{i}_{d \text { peak }}\left(\frac{1}{\pi}+\frac{1}{2} \sin \omega_{0} t-\frac{2}{\pi} \sum_{n=2,4, \ldots}^{\infty} \frac{1}{n^{2}-1} \cos n \omega_{0} t\right)
$$




$$
\mathbf{i}_{d 2}=\mathbf{i}_{d \text { peak }}\left(\frac{1}{\pi}-\frac{1}{2} \sin \omega_{0} t-\frac{2}{\pi} \sum_{n=2,4, \cdots}^{\infty} \frac{1}{n^{2}-1} \cos n \omega_{0} t\right)
$$

This shows that the fundamental components are $180^{\circ}$ out-ofphase and harmonics are in-phase. A multioctave $180^{\circ}$ hybrid is used to combine the fundamental components at the $\Sigma$ port. Harmonics are dissipated through the load at the $\Delta$ port.

The architecture of the newly proposed active integratedantenna push-pull PA is shown in Fig. 1(b). The input stage is identical to that of the conventional microwave frequency push-pull, consisting of a $180^{\circ}$ hybrid to provide the proper waveforms to the MESFET transistors. However, a multifeed planar antenna has replaced the output hybrid. For proper operation, feeding must be done in such a manner that desired radiation mode is excited $180^{\circ}$ out-of-phase by each feed. As will be discussed later, a number of planar antennas are amenable for this type of architecture. The first reported integrated-antenna PA used a dual-feed patch antenna with feeds placed at opposite radiating edges [12], [13].

A crucial issue for this architecture is treatment of higher harmonics. For high-efficiency operation, the load impedance should provide a reactive termination at higher harmonics to reflect the power back to the MESFET with proper phase. Ideally, a short circuit should be placed at the drain of the device for proper harmonic termination. However, at microwave frequencies, due to the device parasitics, the second harmonic is reflected back to the FET with the appropriate phase to resonate the drain-to-source capacitance. Therefore, the output circuit should have magnitude of the reflection coefficient of 1.0 at the second harmonic and appropriate phase chosen to maximize efficiency.

\section{Dual-FeEd Planar Antennas}

Dual-feed antennas can be described as a three-port structure, where ports 1 and 2 are the antenna feeds, and port 3 is an invisible radiation port.

Three conditions must exist if the two-feed antenna is to be used as an efficient power combiner in a tuned push-pull PA. First, the antenna must radiate efficiently with acceptable patterns. Second, the input impedance of the antenna should be suitable for harmonic tuning. Finally, negligible power should flow from feed port to feed port when push-pull excitation is applied, which will directly limit the power-combining efficiency. With two specific examples, we will show that all conditions can be met and that the power-combining efficiency will approach $100 \%$.

Two structures that satisfy these conditions are the microstrip patch and slot antennas, shown in Fig. 2(a) and (b), along with their radiation mode profiles. The patch antenna has microstrip feeds placed at opposite radiating edges to excite the proper radiation mode. The $\lambda$-long slot uses two microstrip lines oriented in opposite directions and placed $\lambda / 2$ apart on the slot. The microstrip feeds of the slot extend $\lambda / 4$ across the slot to form a virtual short circuit for strong coupling to the antenna.
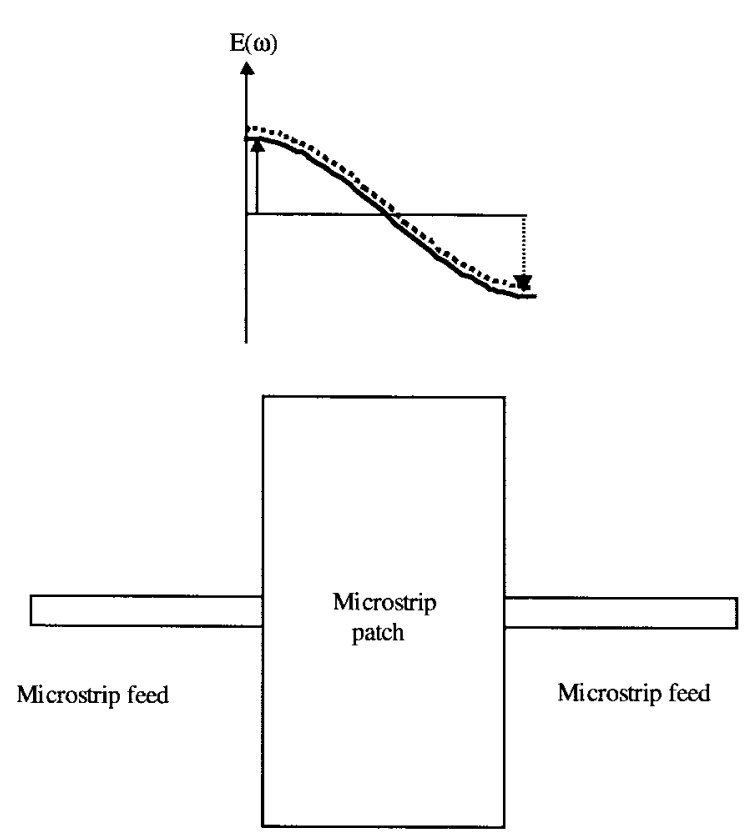

(a)

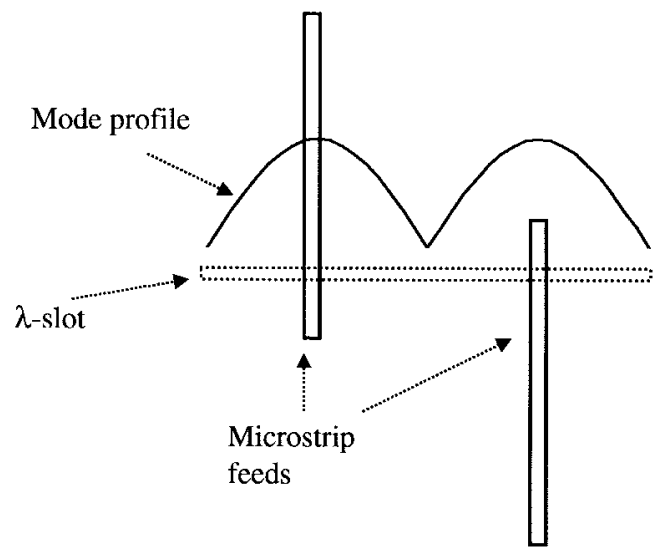

(b)

Fig. 2. Two dual-feed antenna structures and mode profile including: (a) dual-feed microstrip patch and (b) dual-feed slot antenna.

For each of these structures used with push-pull excitation, each feed of the structure will excite identical radiation modes with identical phase in the antenna. Therefore, the radiated power will combine in free space. Additionally, the radiated fields will be essentially that of the single-feed patch or slot antenna. This has been confirmed by measurements in an anechoic chamber. Therefore, each of these structures satisfies the first condition. Fig. 3(a) and (b) shows the radiation patterns and gain of the slot antenna.

Each of these structures can also be used for harmonic tuning. Measured $S$-parameters are shown in Fig. 4(a) and (b). Note that $S_{21}$ represents the feed-feed coupling and can be quite high for single-feed excitation (later we will show that it is ideally zero for push-pull excitation). For harmonic tuning, the magnitude of $S_{11}$ should be close to $0 \mathrm{~dB}$ at harmonic frequencies. From Fig. 4(a), the dual-feed patch antenna can perform second harmonic tuning if $f_{0}$ is chosen at $2.5 \mathrm{GHz}$. Additionally, Fig. 4(b) shows that the dual-feed slot antenna 


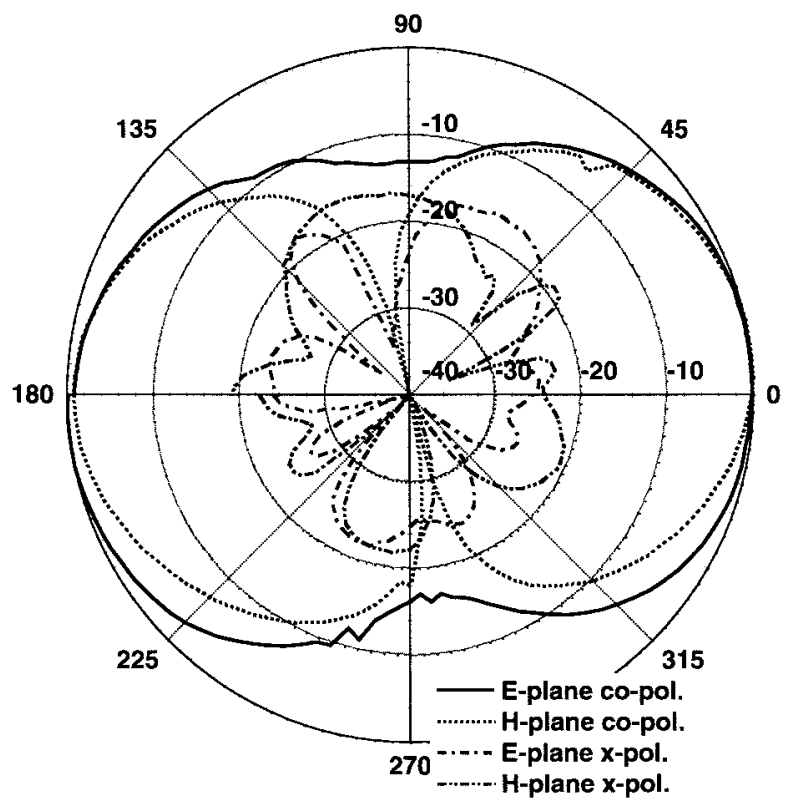

(a)

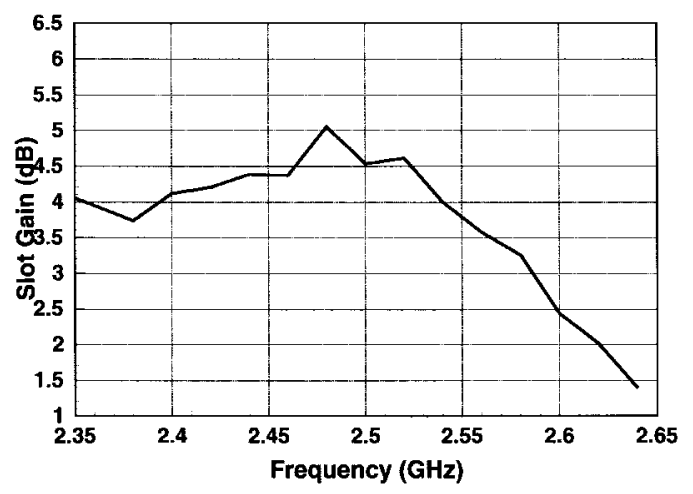

(b)

Fig. 3. Radiation characteristics of dual-feed slot antenna including: (a) radiation patterns at $2.42 \mathrm{GHz}$ and (b) measured antenna gain as a function of frequency.

can perform both second and fourth harmonic tuning if $f_{0}$ is chosen at $2.3 \mathrm{GHz}$. This is because the $\lambda / 4$ stub used to couple the fundamental signal to the slot will appear as an open circuit at even harmonics. In fact, the input impedance at the second and fourth harmonics are 6000 and $1000 \Omega$, respectively.

Finally, the feed-feed coupling of each of these structures is ideally zero when push-pull excitation is applied. First, consider the patch antenna. In this case, the mode coupling between feeds 1 and 2 will be the microstrip mode. With push-pull excitation (odd mode) applied at each feed, the voltage at the center of the patch must be zero. Therefore, a virtual short circuit exists at the center of the patch and no net power will be guided between feeds 1 and 2 . This creates a standing wave in the cavity that exactly corresponds to the desired radiation mode of the fundamental resonance shown in Fig. 2(a). Therefore, any propagating energy will be directly transformed into the radiation mode.

Similar results are true for the slot antenna. In this case, the propagating mode between feeds 1 and 2 will be the slotline mode. However, due to the polarization of the slotline mode,

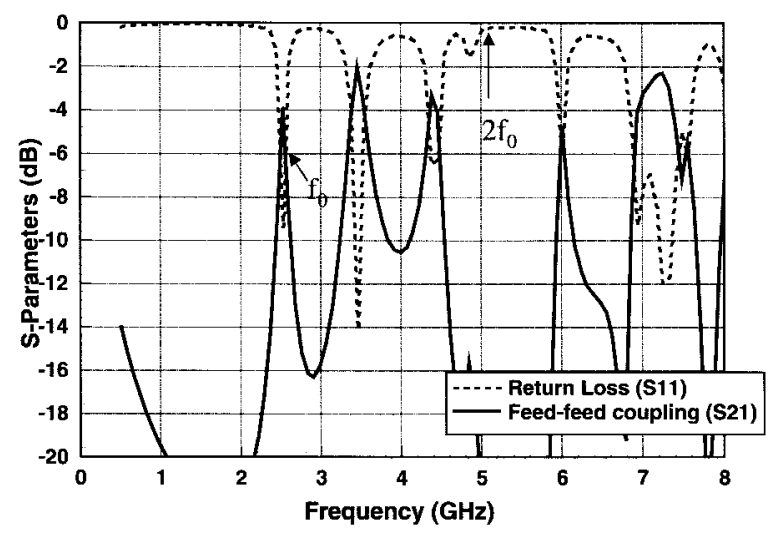

(a)

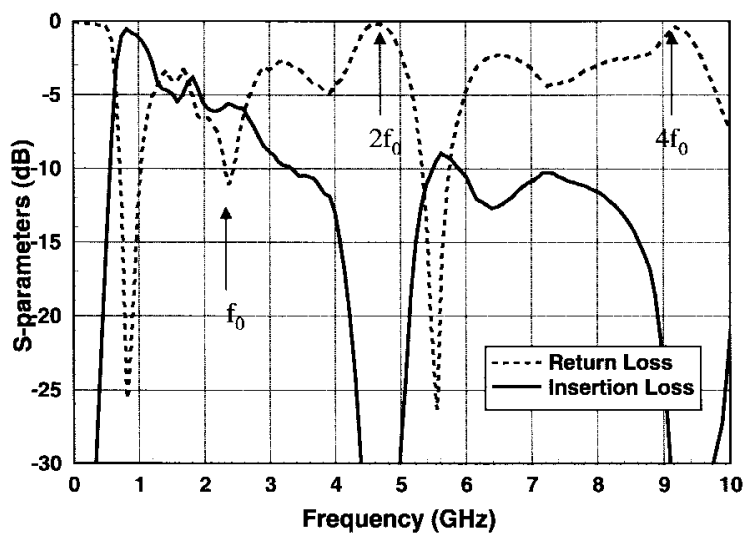

(b)

Fig. 4. Measured $S$-parameters of: (a) dual-feed patch antenna and (b) dual-feed slot antenna.

when push-pull excitation is applied to the oppositely oriented microstrip feeds, even excitation will occur. Therefore, zero current will flow across the middle of the slot antenna and a virtual open-circuit exists. Again, no power will flow between the feed ports. Since no power will flow between the two feed ports, only antenna losses will limit the power-combining efficiency. In the case of small antenna losses, the combing efficiency will, therefore, approach $100 \%$.

An additional comment should be made about the bandwidth of the two structures. The bandwidth of the patch antenna is quite narrow and will limit the overall system bandwidth. The slot is considerably more broadband than the patch.

As we will see, either can be used effectively in a push-pull PA. Size, radiation patterns, and bandwidth will determine choice between the two.

\section{PA RESULTS}

Push-pull PA's using each dual-feed antenna have been designed and tested. They were designed with HP-Series IV. The dual-feed antenna is incorporated directly into the simulation as a two-port network using experimental $S$-parameter data. The device used is a Microwave Technology MWT-8HP GaAs FET. For maximum PAE, each amplifier is biased as Class $\mathrm{AB}$, where they have slightly higher gain. A nonlinear device model and harmonic balance are used in simulation. 


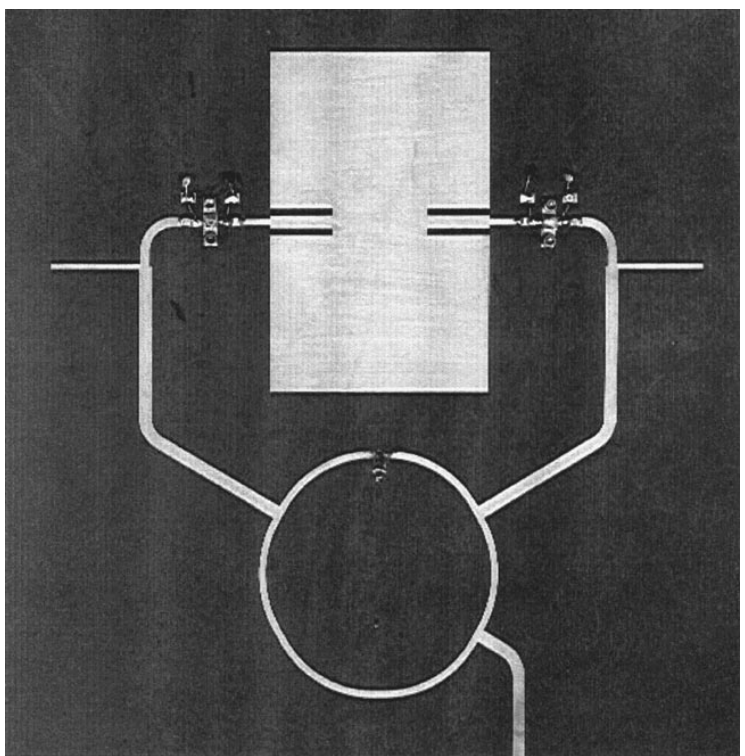

Fig. 5. Photograph of patch-antenna push-pull PA.

Systematic measurement of integrated-antenna amplifiers is complicated by the fact that the output load is free space rather than a $50-\Omega$ load. For a fair amplifier comparison, the gain of the antenna must be calibrated out of the measurement. The simplest way to do this is to use a passive version of the integrated-antenna amplifier as a standard. In this technique, the power radiated from the active antenna is measured at a convenient angle (usually at broadside). The passive circuit is then substituted and the measurement is repeated. With the same input power and compensating for any mismatch losses of the passive standard, the gain of the amplifier is the difference between the two measurements. This procedure assumes that the radiation patterns for the active and passive antennas are identical. This has been confirmed by measuring the radiation patterns of both the active and passive versions of the antennas, which are virtually the same.

\section{A. Amplifier Integrated with a Patch Antenna}

The circuit is designed for operation around $2.5 \mathrm{GHz}$. The completed circuit is shown in Fig. 5. We can see one of the benefits of the integrated-antenna push-pull PA from the figure. Most of the circuit space is used by the antenna and input hybrid. If we had not used the dual-feed patch antenna and included an output hybrid, circuit size would be approximately $30 \%$ larger.

Figs. 6 and 7 show simulated and measured PAE and output power versus input power. Agreement between experimental and measurement are good. Maximum measured PAE is $55 \%$ at an output power of $25 \mathrm{dBm}$.

Of additional interest is the harmonic radiation of the amplifier, which has been shown in [12]. Experimentally, second harmonic suppression is estimated by integrating the $E$ - and $H$-plane patterns of the fundamental and second harmonic and is found to be approximately $17 \mathrm{~dB}$, where the Friis transmission formula is used to calibrate the radiated power. Higher harmonics were quite low and were not examined in detail.

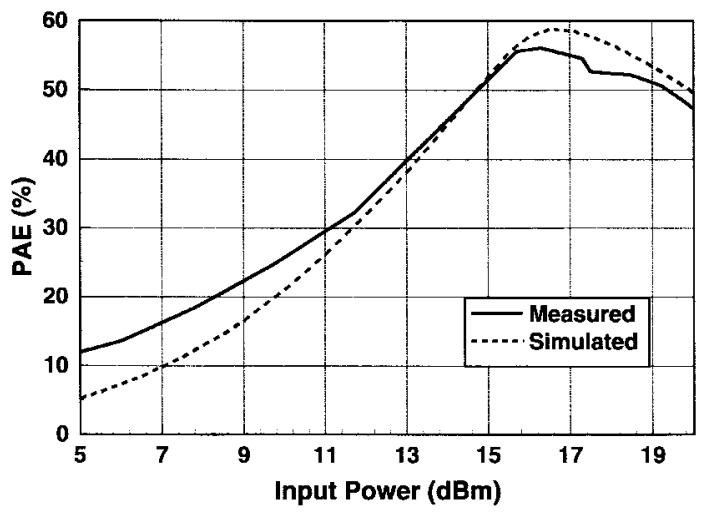

Fig. 6. Simulation and measurement of PAE versus input power for the PA integrated with a patch antenna. The operating frequency is $2.5 \mathrm{GHz}$.

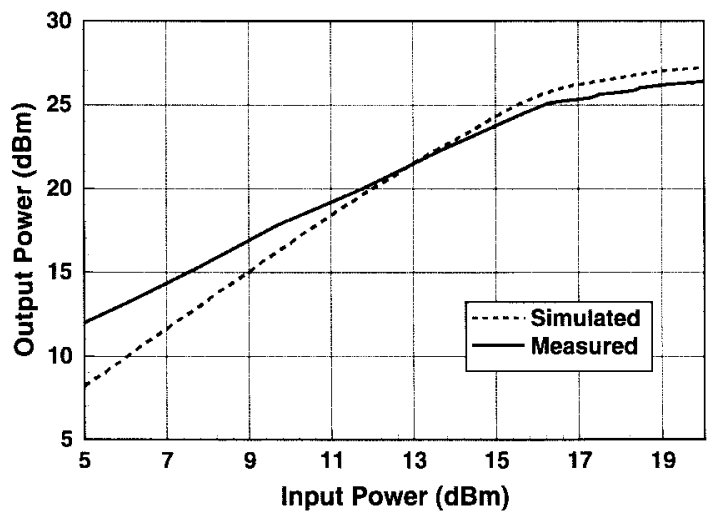

Fig. 7. Simulation and measurement of output power versus input power for the PA integrated with a patch antenna. The operating frequency is $2.5 \mathrm{GHz}$.

\section{B. Amplifier Integrated with a Slot Antenna}

Fig. 8(a) and (b) shows the completed slot antenna push-pull PA and the reference slot antenna used as a standard. Note that circuit size has been further reduced by using a Wilkinson power divider and a $180^{\circ}$ delay line to provide antiphase excitation to the push-pull, which is more compact than a $180^{\circ}$ hybrid. This could have also been applied to the patch version with a further reduction of approximately $30 \%$ for that circuit.

Figs. 9 and 10 show PAE and output power versus input power for an operating frequency of $2.46 \mathrm{GHz}$. There is excellent agreement between simulation and measurement in both cases. Maximum measured PAE is $63 \%$ at an output power of $26 \mathrm{dBm}$. PAE is increased over the patch version because the load is fully tuned and fourth harmonic power has also been converted to the fundamental. Fig. 11 shows simulated and measured dc drain currents for the two FET's. The increase in average drain current is expected for class-AB operation. Ideally, two currents should be identical. Deviation between the two current characteristics is due to mismatch between the devices. Figs. 12 and 13 show PAE and output power versus frequency. PAE is better than $55 \%$ from 2.43 to $2.61 \mathrm{GHz}$, which is $8 \%$ bandwidth better than $55 \%$ PAE. 


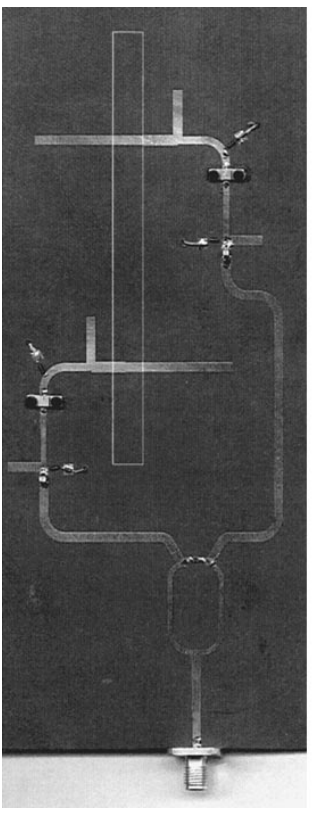

(a)

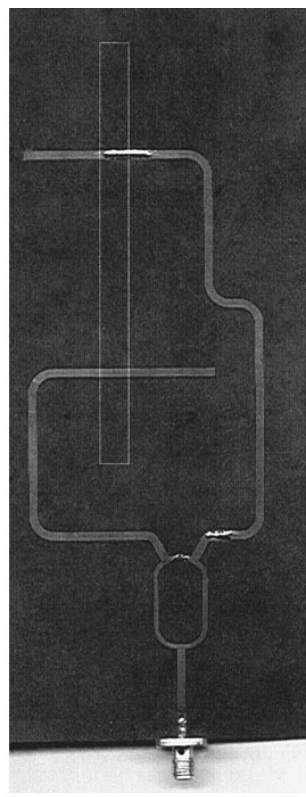

(b)
Fig. 8. Photograph of: (a) slot antenna push-pull PA and (b) reference antenna used for calibration. The slot antenna is etched in the lower ground and is represented by a white outline.

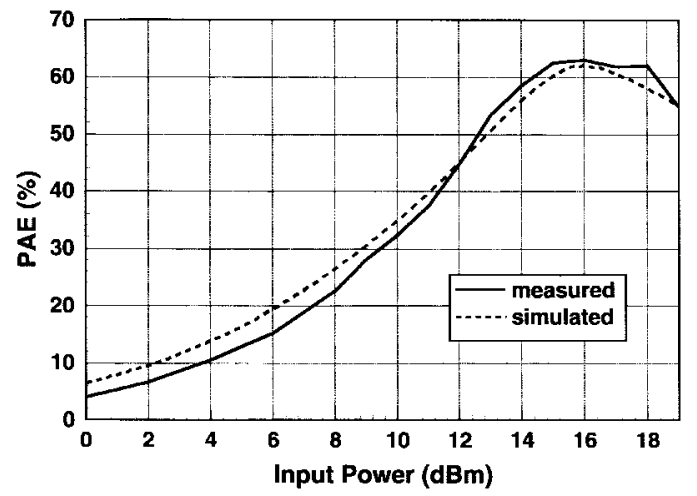

Fig. 9. Simulation and measurement of PAE versus input power for the PA integrated with a slot antenna. The operating frequency is $2.46 \mathrm{GHz}$.

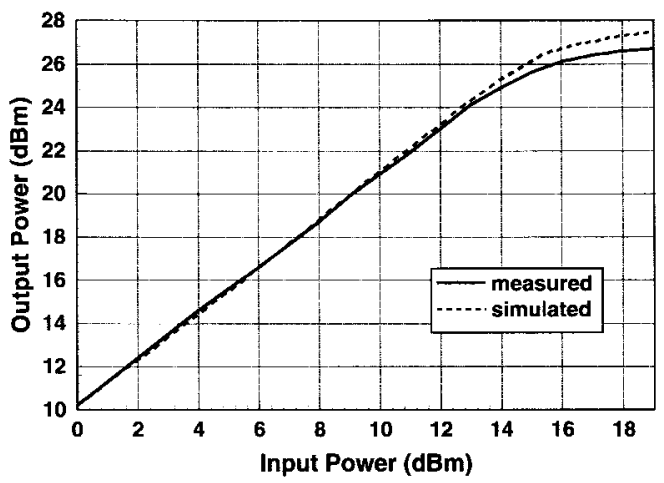

Fig. 10. Simulation and measurement of output power versus input power for the PA integrated with slot antenna. The operating frequency is $2.46 \mathrm{GHz}$.

The harmonic tuning technique presented here cannot only improve the amplifier efficiency, but can also reduce unwanted harmonic radiation. This is observed by measuring the second

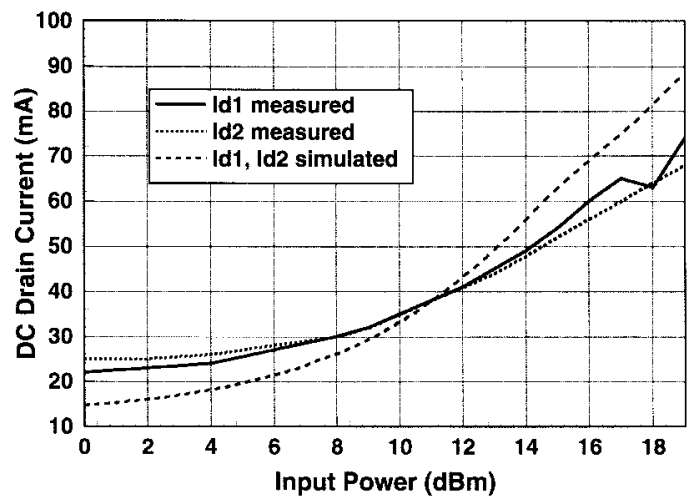

Fig. 11. Simulation and measurement of de drain currents for two FET's versus input power for the PA integrated with slot antenna. The operating frequency is $2.46 \mathrm{GHz}$.

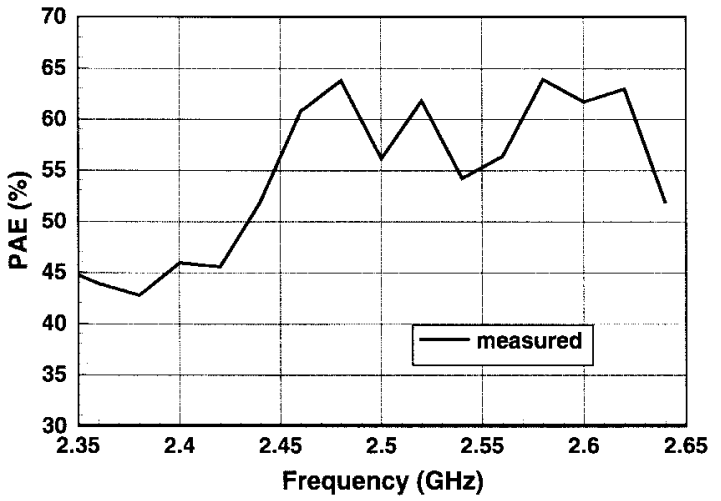

Fig. 12. Measured PAE versus frequency for the PA integrated with slot antenna.

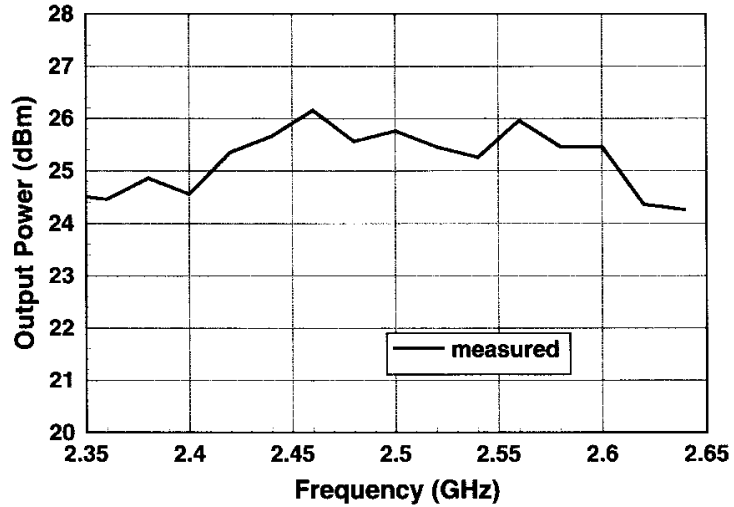

Fig. 13. Measured output power versus frequency for the PA integrated with a slot antenna.

and third harmonic radiation from the slot push-pull amplifier. Since harmonic frequencies typically have different radiation patterns from that of the fundamental, the harmonic radiated power has to be measured in all directions, and is shown in Fig. 14(a) and (b). The second harmonic is below $-33 \mathrm{~dB}$ in all directions for each polarization. The third harmonic is below $-30 \mathrm{~dB}$ in all directions for each polarization. Both results are measured at maximum PAE. Note that the fundamental 


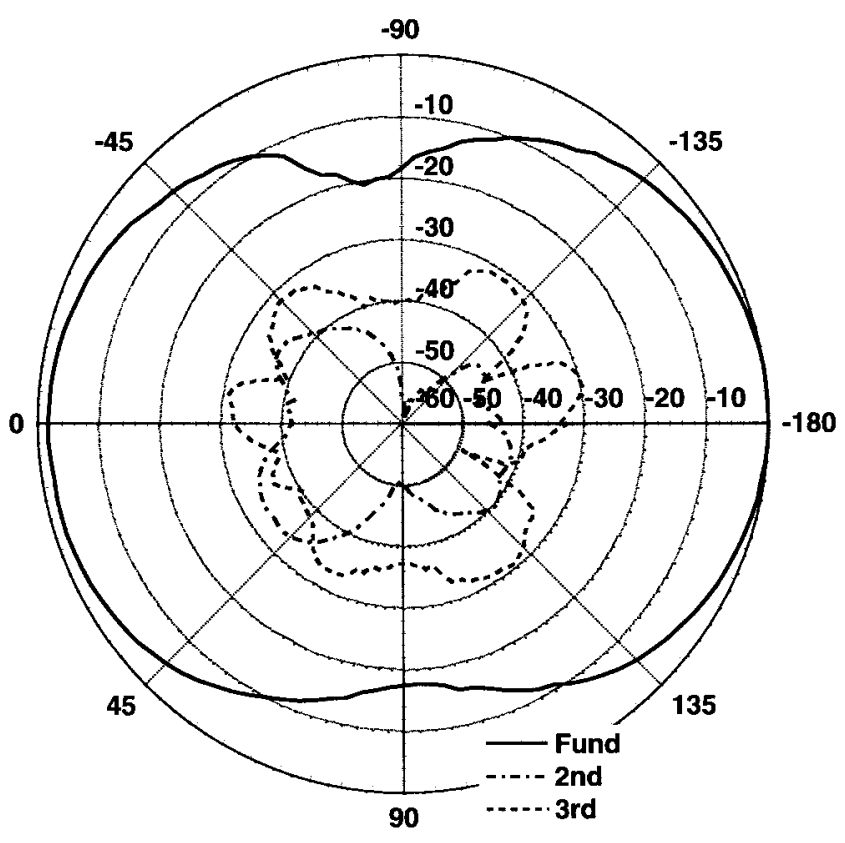

(a)

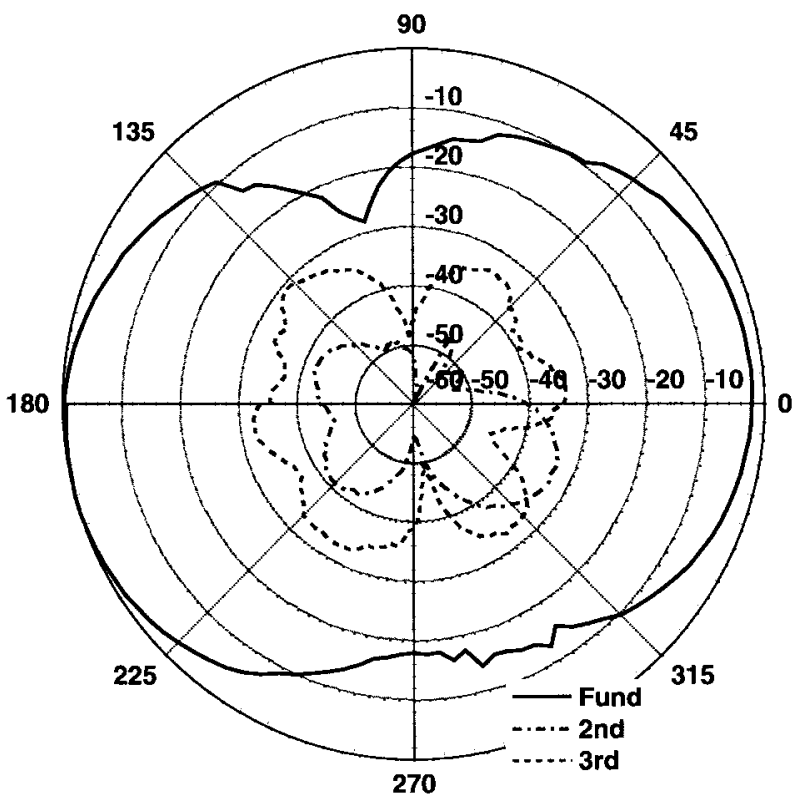

(b)

Fig. 14. Measured fundamental, second, and third harmonic: (a) $E$ - and (b) $H$-radiation patterns for the PA integrated with a slot antenna.

has been normalized to $0 \mathrm{~dB}$ and higher harmonics have been calibrated to the fundamental using the Friis transmission formula.

In digital wireless systems, amplifiers are seldom operated at maximum efficiency due to distortion caused by nonlinearity. Instead, efficiency will be compromised as the amplifier is "backed-off" to a level where the distortion can be tolerated.

This is illustrated by testing amplifier performance with a CDMA input signal. In this case, the maximum operating point is determined where the ACPR is better than $-42 \mathrm{dBc}$ at a $650-\mathrm{kHz}$ offset, which is more stringent than the IS98 linearity
TABLE I

Frequency, InPut and Output Power and PAE for Which Slot Amplifier LiNEARITY Is BETTER THAN $-42 \mathrm{dBc}$ AT A $650-\mathrm{kHz}$ OFFSET

\begin{tabular}{l|l|l|l}
\hline $\begin{array}{l}\text { Frequency } \\
(\mathrm{GHz})\end{array}$ & $\begin{array}{l}\text { Input } \\
\text { Power } \\
(\mathrm{dBm})\end{array}$ & $\begin{array}{l}\text { Output } \\
\text { Power } \\
(\mathrm{dBm})\end{array}$ & $\begin{array}{l}\text { PAE } \\
\text { (percent) }\end{array}$ \\
\hline 2.38 & 10.7 & 21.6 & 33 \\
\hline 2.40 & 11.7 & 21.3 & 32 \\
\hline 2.42 & 11.2 & 22.2 & 38 \\
\hline 2.44 & 12.7 & 22.9 & 43 \\
\hline 2.46 & 12.2 & 23.4 & 48 \\
\hline 2.48 & 12.2 & 22.5 & 39 \\
\hline 2.50 & 12.7 & 23.0 & 42 \\
\hline 2.52 & 12.2 & 22.6 & 38 \\
\hline 2.54 & 13.2 & 22.8 & 40 \\
\hline 2.56 & 12.2 & 23.2 & 47 \\
\hline 2.58 & 12.7 & 22.9 & 45 \\
\hline 2.60 & 11.7 & 22.7 & 46 \\
\hline
\end{tabular}

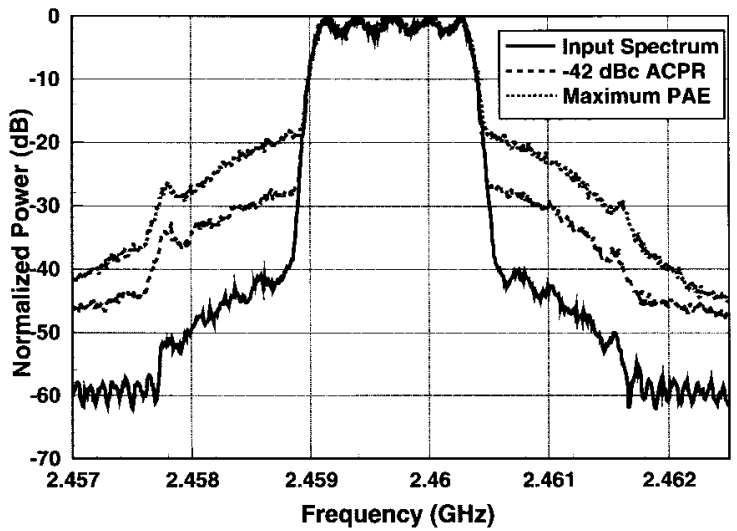

Fig. 15. CDMA power spectrums for the slot push-pull PA. Shown are the CDMA input signal, and output spectrums for when the amplifier is backed off to meet the $-42-\mathrm{dBc}$ ACPR specification and for maximum power added efficiency.

specification. As can be seen from Table I, the linearity of the amplifier is quite good. The maximum PAE for which the amplifier passes this spec reaches $48 \%$. Effects of nonlinearity are clear in Fig. 15. The CDMA input signal is shown, along with normalized output spectrum for the amplifier operated at $-42-\mathrm{dBc}$ ACPR $\left(P_{\mathrm{in}}=12.2 \mathrm{dBm}\right)$ and operated at maximum efficiency $\left(P_{\mathrm{in}}=16 \mathrm{dBm}\right)$.

\section{CONCLUSION}

In this paper, two active integrated-antenna versions of the push-pull PA have been presented. This topology utilizes a dual-feed planar antenna as a $180^{\circ}$ hybrid and radiator, therefore eliminating the losses of the output hybrid and reducing transmitter size. As seen with two design examples, this topology has demonstrated high-efficiency performance and excellent linearity.

Two designs have been presented. The first consists of a dual-feed patch-antenna version of the push-pull PA. Maximum PAE of $55 \%$ efficiency has been demonstrated at the operating frequency of $2.5 \mathrm{GHz}$. While this design is compact and does give good performance, bandwidth of the amplifier is small (limited by the bandwidth of the patch antenna) 
and no direct physical mechanism exists for harmonic tuning other than simply mismatching the antenna at harmonic frequencies.

The second design consists of a dual-feed version of the slot antenna integrated with the PA. This structure is considerably more broad-band (demonstrates PAE greater than 55\% over an $8 \%$ bandwidth) and has excellent properties for harmonic tuning of the second and fourth harmonics. Radiation patterns show that the higher harmonics have been successfully suppressed. Additionally, harmonic tuning has increased the maximum PAE to $63 \%$. The circuit has also been tested for CDMA performance and demonstrates excellent linearity. The dual-feed slot-antenna push-pull PA achieves better than $-42-\mathrm{dBc}$ ACPR when backed off to an operating point that yields $48 \%$ PAE.

On additional comment should be made about the powercombining efficiency of this approach. The results of this paper can be compared with the tuned single-ended integratedantenna amplifiers presented in [10], which used the identical FET transistor at a comparable frequency. Maximum PAE results using our approach are virtually identical and probably approach the maximum for this device, which is a function of device gain. From this observation, we can conclude that the power-combining capability of the dual-feed antenna approaches $100 \%$. Additionally, the power output at maximum PAE is also increased.

\section{ACKNOWLEDGMENT}

The authors greatly appreciate the help that Dr. C. Y. Lee rendered for this paper.

\section{REFERENCES}

[1] J. L. B. Walker, High-Power GaAs FET Amplifier. Norwood: MA, Artech House, 1993.

[2] P.-C. Hsu, C. Nguyen, and M. Kintis, "Uniplanar broad-band push-pull FET amplifiers," IEEE Trans. Microwave Theory Tech., vol. 45, pp. 2150-2151, Dec. 1997.

[3] S. Toyoda, "High efficiency amplifiers," in IEEE MTT-S Symp. Dig., 1994, pp. 253-256.

[4] E. A. Sovero, J. B. Hacker, J. A. Higgins, D. S. Deakin, and A. L. Sailer, "A $\mathrm{Ka}$-band monolithic quasi-optic amplifier," in IEEE MTT-S Symp. Dig., 1998, pp. 1453-1456.

[5] T. B. Mader, E. W. Bryerton, M. Marckovic, M. Forman, and Z. Popović, "Switched-mode high efficiency microwave power amplifiers in a free-space power combining array," in IEEE MTT-S Symp. Dig., 1998, pp. 1453-1456.

[6] J. B. Hacker, R. M. Weikle II, M. Kim, M. P. DeLisio, and D. B. Rutledge, "A 100-element planar Schottky diode grid mixer," IEEE Trans. Microwave Theory Tech., vol. 40, pp. 557-562, Mar. 1992.

[7] B. K. Kormanyos, P. H. Ostdiek, W. L. Bishop, T. W. Crowe, and G. M Rebeiz, "A planar wide-band 80-200-GHz subharmonic receiver," IEEE Trans. on Microwave Theory Tech., vol. 41, pp. 1730-1737, Oct. 1993.

[8] D. Singh, P. Gardner, and P. S. Hall, "Frequency doubling integrated push-push microstrip transponder," in European Microwave Conf. Dig., 1997, pp. 1181-1185.

[9] V. Radisic, S. T. Chew, Y. Qian, and T. Itoh, "High-efficiency power amplifier integrated with antenna," IEEE Microwave Guided Wave Lett., vol. 7, pp. 39-41, Feb. 1997.

[10] V. Radisic, Y. Qian, and T. Itoh, "Novel architectures for high efficiency amplifiers for wireless applications," IEEE Trans. Microwave Theory Tech., vol. 46, , pp. 1901-1909, Nov. 1998.

[11] J. Lin and T. Itoh, "Active integrated antennas," IEEE Trans. Microwave Theory Tech., vol. 42, pp. 2186-2194, Dec. 1994.
[12] W. R. Deal, V. Radisic, Y. Qian, and T. Itoh, "Novel push-pull integrated antenna transmitter front-end," IEEE Microwave Guided Wave Lett., vol. 8, pp. 405-407, Nov. 1998

[13] _ "Power measurements for push-pull integrated antenna power combiner," in Asia-Pacific Microwave Conf. Dig., 1998, pp.

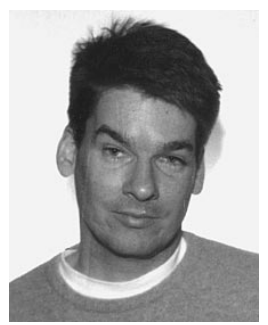

William R. Deal (S'85-M'85) received the B.S degree in electrical engineering from the University of Virginia, Charlottesville, in 1996, the M.S. degree in electrical engineering from the University of California at Los Angeles (UCLA), in 1998, and is currently working toward the Ph.D. degree at UCLA.

His research interests include planar antennas, quasi-optics, integrated-antenna technology, and power-amplifier design.

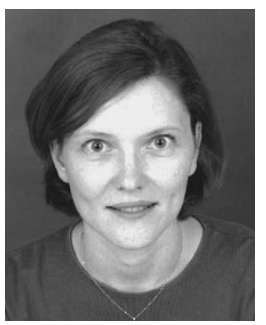

Vesna Radisic (S'91-M'93) received the Dipl.Ing. degree in electrical engineering from the University of Belgrade, Belgrade, Yugoslavia in 1991, the M.S.E.E. degree from the University of Colorado at Boulder, in 1993, and the Ph.D. degree in electrical engineering from the University of California at Los Angeles, in 1998.

From 1994 to 1995, she was with the Ortel Corporation, where she was involved with broadband RF/fiber-optic transmitters and receivers. She is currently with HRL Laboratories, Malibu, CA where she is working on monolithic-microwave integrated-circuit (MMIC) design and device modeling.

Ms. Radisic received third place in the 1998 Student Paper Competition presented at the IEEE International Microwave Symposium.

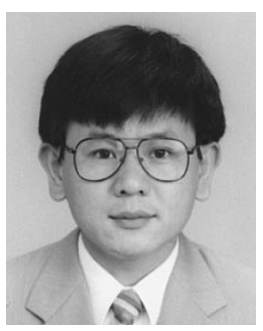

Yongxi Qian (S'91-M'93) was born in Shanghai, China, in 1965. He received the B.E. degree from Tsinghua University, Beijing, China, in 1987, and the M.E. and Ph.D. degrees from the University of Electro-Communications, Tokyo, Japan, in 1990 and 1993, respectively, all in electrical engineering.

From 1993 to 1996, he was an Assistant Professor at the University of Electro-Communications, Tokyo, Japan. He joined the University of California at Los Angeles, in April 1996, and is currently an Assistant Research Engineer in the Electrical Engineering Department. He has been involved with various numerical techniques for microwave and millimeter-wave circuits and antennas, generation and transmission of picosecond electrical pulses, crosstalk problems in high-density MMIC's, miniature circuits for mobile communications, and millimeter-wave focal plane imaging arrays. $\mathrm{He}$ has authored or coauthored over 110 refereed journal and conference papers, two book chapters, and one book. His current research interests include broad-band planar antennas, reconfigurable multiband/multifunction apertures (RECAP's), RF interconnect for mixed-signal silicon MMIC's, quasi-optical power combining, photonic bandgap (PBG) structures, high-efficiency microwave amplifiers, active integrated antennas for multimedia communication systems, and highpower broad-band RF photonic devices for millimeter and submillimeter-wave photomixing.

Dr. Qian is the recipient of the 1998 Japan Microwave Prize presented at the Asia-Pacific Microwave Conference. 


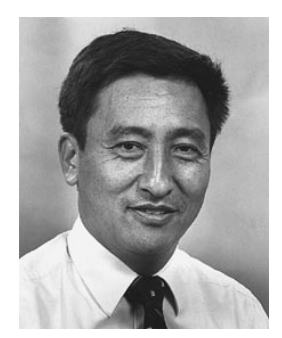

Tatsuo Itoh (S'69-M'69-SM'74-F'82-LF'94) received the Ph.D. Degree in electrical engineering from the University of Illinois at Urbana-Champaign, in 1969.

From September 1966 to April 1976, he was with the Electrical Engineering Department, University of Illinois at Urbana-Champaign From April 1976 to August 1977, he was a Senior Research Engineer in the Radio Physics Laboratory, SRI International, Menlo Park, CA. From August 1977 to June 1978, he was an Associate Professor at the University of Kentucky, Lexington. In July 1978, he joined the faculty at The University of Texas at Austin, where he became a Professor of electrical engineering in 1981 and Director of the Electrical Engineering Research Laboratory in 1984. During the summer of 1979, he was a Guest Researcher at AEG-Telefunken, Ulm, Germany. In September 1983, he was selected to hold the Hayden Head Centennial Professorship of Engineering at The University of Texas at Austin. In September 1984, he was appointed Associate Chairman for Research and Planning of the Electrical and Computer Engineering Department, The University of Texas at Austin. In January 1991, he joined the University of California at Los Angeles, as Professor of electrical engineering and holder of the TRW Endowed Chair in Microwave and Millimeter Wave Electronics.
He was an Honorary Visiting Professor at Nanjing Institute of Technology, China, and the Japan Defense Academy. In April 1994, he was appointed as Adjunct Research Officer for the Communications Research Laboratory, Ministry of Post and Telecommunication, Japan. He currently holds a Visiting Professorship at The University of Leeds, Leeds, U.K., and is an External Examiner of the Graduate Program of City University of Hong Kong, Hong Kong. He has authored or co-authored over 250 journal publications, 475 refereed conference presentations, and over 26 books/book chapters in the area of microwaves, millimeter-waves, antennas and numerical electromagnetics. He has also produced $42 \mathrm{Ph}$.D. students. He was the chairman of USNC/URSI Commission D from 1988 to 1990, the vice chairman of Commission D of the International URSI for 1991-1993 and chairman of the same Commission for 1993-1996. He is on Long Range Planning Committee of URSI. He serves on advisory boards and committees of a number of organizations.

Dr. Itoh is a member of the Institute of Electronics and Communication Engineers of Japan, and Commissions B and D of USNC/URSI. He served as the Editor-in-Chief of the IEEE Transactions on Microwave THeORY AND TeChNiques from 1983 to 1985 and of the IEEE Microwave AND Guided WAVE LETTERS from 1991 to 1994. He serves on the Administrative Committee of IEEE Microwave Theory and Techniques Society (MTT-S). He was vice president of the IEEE MTT-S in 1989 and president in 1990. He has received a number of awards, including the 1998 Shida Award from the Japanese Ministry of Post and Telecommunications and the 1998 Japan Microwave Prize. 